\title{
Ground-State Spin Blockade in a Single-Molecule Junction
}

de Bruijckere, J.; Gehring, P.; Palacios-Corella, M.; Clemente-Leon, M.; Coronado, E.; Paaske, J.; Hedegard, P.; van der Zant, H. S. J.; Hedegård, Per

Published in:

Physical Review Letters

DOI:

10.1103/PhysRevLett.122.197701

Publication date:

2019

Document version

Publisher's PDF, also known as Version of record

Citation for published version (APA):

de Bruijckere, J., Gehring, P., Palacios-Corella, M., Clemente-Leon, M., Coronado, E., Paaske, J., Hedegard, P., van der Zant, H. S. J., \& Hedegård, P. (2019). Ground-State Spin Blockade in a Single-Molecule Junction. Physical Review Letters, 122(19), [197701]. https://doi.org/10.1103/PhysRevLett.122.197701 


\title{
Ground-State Spin Blockade in a Single-Molecule Junction
}

\author{
J. de Bruijckere, ${ }^{1}$ P. Gehring, ${ }^{1}$ M. Palacios-Corella, ${ }^{2}$ M. Clemente-León, ${ }^{2}$ E. Coronado, ${ }^{2}$ J. Paaske,,${ }^{3,4}$ \\ P. Hedegård, ${ }^{3}$ and H. S. J. van der Zant ${ }^{1, *}$ \\ ${ }^{1}$ Kavli Institute of Nanoscience, Delft University of Technology, 2628 CJ Delft. The Netherlands \\ ${ }^{2}$ Instituto de Ciencia Molecular (ICMol), Universidad de Valencia, Catedrático José Beltrán 2, Paterna, 46980, Spain \\ ${ }^{3}$ Niels Bohr Institute, University of Copenhagen, DK-2100 Copenhagen, Denmark \\ ${ }^{4}$ Center for Quantum Devices, Niels Bohr Institute, University of Copenhagen, DK-2100 Copenhagen, Denmark
}

(Received 4 December 2018; revised manuscript received 21 February 2019; published 14 May 2019)

\begin{abstract}
It is known that the quantum mechanical ground state of a nanoscale junction has a significant impact on its electrical transport properties. This becomes particularly important in transistors consisting of a single molecule. Because of strong electron-electron interactions and the possibility of accessing ground states with high spins, these systems are eligible hosts of a current-blockade phenomenon called a ground-state spin blockade. This effect arises from the inability of a charge carrier to account for the spin difference required to enter the junction, as that process would violate the spin selection rules. Here, we present a direct experimental demonstration of a ground-state spin blockade in a high-spin single-molecule transistor. The measured transport characteristics of this device exhibit a complete suppression of resonant transport due to a ground-state spin difference of $3 / 2$ between subsequent charge states. Strikingly, the blockade can be reversibly lifted by driving the system through a magnetic ground-state transition in one charge state, using the tunability offered by both magnetic and electric fields.
\end{abstract}

DOI: 10.1103/PhysRevLett.122.197701

Quantum transport through nano-objects is an important research direction comprising different phenomena, some of which could potentially be used for spintronics [1-3] and quantum computing [4-6] applications. Intriguing examples of quantum transport phenomena include blockades of electric current. Various physical phenomena are involved in the underlying mechanisms of these blockades: the current in double quantum dots can be inhibited by the Pauli exclusion principle [7-9], vibrational transitions in molecular junctions and quantum dots can be suppressed by the Franck-Condon blockade [10-12], and in junctions with superconducting electrodes there may be no lowenergy transport as a result of the superconducting energy gap $[13,14]$. These blockade mechanisms all occur in combination with the Coulomb blockade, which results from the energy level spacing $\Delta E$ and the energy costs of charging a weakly coupled nano-object, i.e., the charging energy $U$. The Coulomb blockade can be overcome as soon as the energy of an electron in one electrode exceeds the addition energy $E_{\text {add }}=\Delta E+U$. Then, electrons can sequentially travel from one electrode to the other via the nano-object. In every sequential electron tunneling (SET) event, the charge of the object changes by an elementary charge and the spin typically by $1 / 2$, due to the added or removed electron.

Here, we study an exceptionally clear manifestation of the blockade phenomenon that occurs when the groundstate spin of subsequent charge states differs by more than $1 / 2$. SET transitions between these ground states are forbidden by the spin selection rules and Coulomb blockade peaks are suppressed [15-17]. We refer to this effect as a ground-state spin blockade (GSSB). One of the necessary requirements for a GSSB is a high-spin ground state, i.e., $S>1$, in one of the charge states. This requirement is hard to obtain for top-down quantum dots and, in the few earlier demonstrations of a GSSB, neither the full suppression of the Coulomb blockade peaks nor control over the blockade was achieved $[18,19]$. For molecules, high-spin ground states can be tailored by chemical design, making them promising candidates for observing a GSSB. In this transport study, we provide experimental evidence for a complete GSSB in a high-spin single-molecule junction. With an external magnetic field, the blockade can be reversibly lifted by driving the molecule in one charge state through a magnetic ground-state transition. The presence of a GSSB puts constraints on the allowed transitions and is used as a diagnostic tool to determine the ground state and excited states of the molecule.

The measurements were carried out with the device sketched in Fig. 1(a). A single molecule is embedded in a circuit with two gold electrodes in which dc current $(I)$ is measured as a function of the applied voltage difference between the electrodes (the bias voltage $V$ ) and the voltage applied to a capacitively coupled gate electrode $\left(V_{g}\right)$. The junction is formed by room-temperature electromigration [20] and self-breaking [21] of a gold nanowire. A dilute solution of the molecules is drop casted on a chip with 24 electromigrated junctions on which, after pumping away 
the solution and cooling down the sample $(T \approx 40 \mathrm{mK})$, three junctions show a Coulomb blockade with addition energies in the typical regime of molecular junctions, i.e., $E_{\text {add }}>100 \mathrm{meV}$. The tunnel couplings and energy level alignments cannot be controlled by this technique and depend on the way the molecule is trapped inside the junction. One of the three samples shows the right combination of a small tunnel coupling, which allows for high-resolution spectroscopy, and a level alignment close to the Fermi level of the electrodes, such that the molecule can be charged within the accessible gatevoltage range. The molecule in the junction is a pyridinefunctionalized $\mathrm{Mn}$ (III) Anderson polyoxometalate [22], which is sketched in Fig. 1(a). Its properties and synthesis are discussed in the Supplemental Material [23].

In Fig. 1(b), the two top panels show differential conductance $(d I / d V)$ maps of the device as a function of $V$ and $V_{g}$, at zero magnetic field and at 8 T. For clarity, we first discuss the map at $8 \mathrm{~T}$, which looks like a common $d I / d V$ map of a Coulomb-blockaded system containing a single object, here, a molecule. The regions labeled $N$ and $N-1$ are regions in which the charge of the molecule is fixed and SET processes are suppressed. Inside these regions, transport is governed by cotunneling (COT) processes, which appear as horizontal lines. The two slanted lines forming the crosslike shape are the edges of the Coulomb diamonds and their point of incidence at zero bias is the charge degeneracy point. The bottom right panel shows that this point appears as a peak in the linearconductance gate trace, i.e., the $d I /\left.d V\right|_{V=0}$ as a function of $V_{g}$. In the top and bottom regions of the $d I / d V$ map, the charge of the molecule can fluctuate, allowing for SET processes to occur. The top SET region contains slanted lines starting from the left Coulomb edge at $V=0.9 \mathrm{mV}$ and, less intense, at $V=0.3 \mathrm{mV}$, both moving towards the top right. These lines correspond to SET processes involving excited states.

Remarkably, in the map at $0 \mathrm{~T}$, the charge degeneracy point is absent: slanted lines are present, but they do not cross at zero bias. At any gate voltage, the linear conductance is suppressed and only SET lines at finite voltages are present. This shows that transitions between the ground state of $N$ and the ground state of $N-1$ are blocked. The complete suppression can be seen more clearly in the zerobias trace presented in the corresponding bottom panel, which in contrast to the $8 \mathrm{~T}$ trace shows no peak. All the slanted lines in the map appear at finite-bias voltages and mark transitions involving at least one excited state: the SET line starting at $V=0.7 \mathrm{mV}$ coincides with a COT line at that same bias voltage, which implies that their excitation energies are equal and involve the same excited state. Similarly, the COT lines at $V= \pm 0.1 \mathrm{mV}$ in $N$ connect to two SET lines starting at the same bias voltages. Another faint COT line appears at $V=-0.5 \mathrm{mV}$ in $N-1$, closer to zero bias than its counterpart at positive
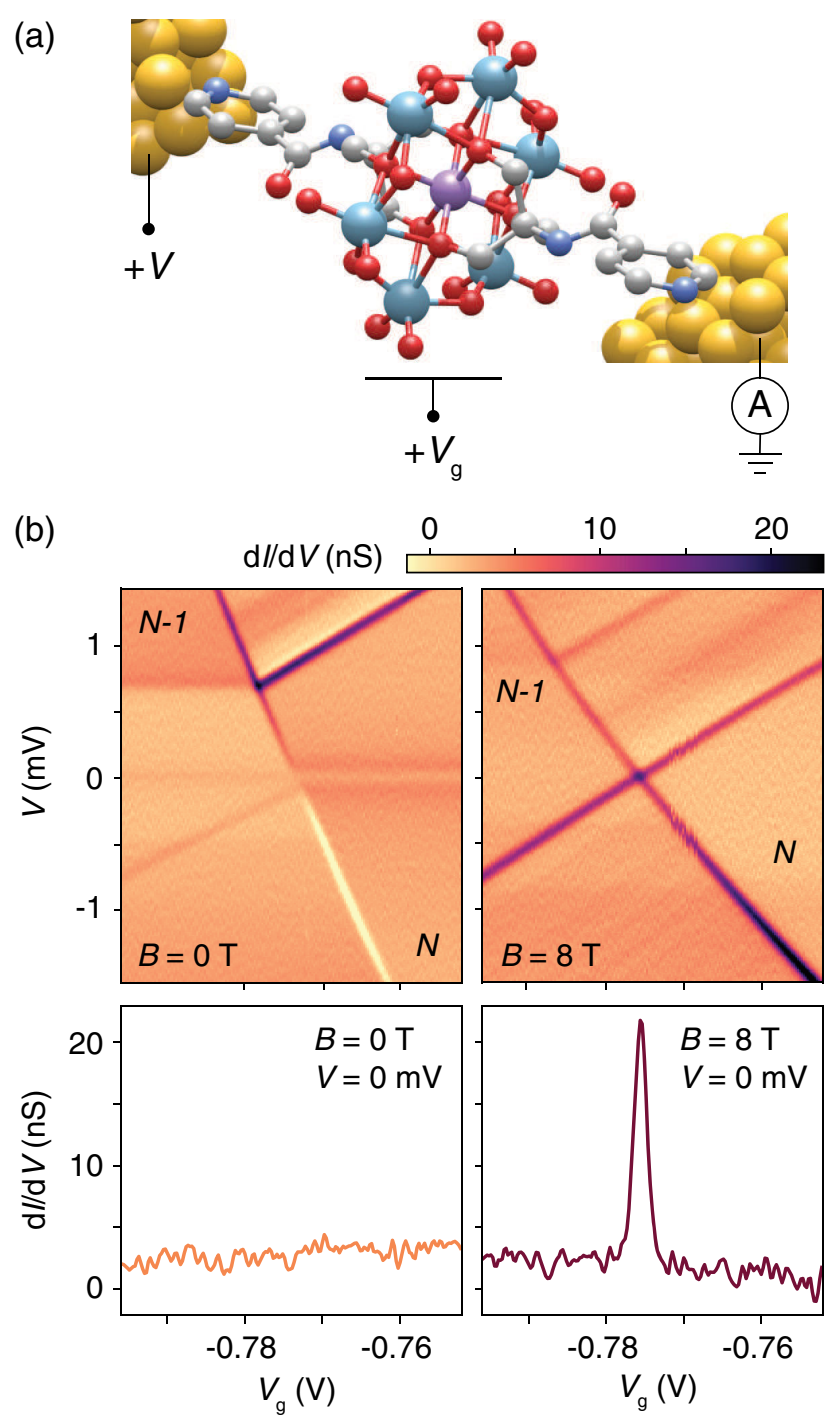

FIG. 1. (a) Sketch of the molecule, gold electrodes, and measurement circuit. The molecule consists of a Mn(III) center (purple) surrounded by six $\mathrm{MoO}_{6}$ octahedra, which connect on both sides to pyridine-based ligands. Electric current $(I)$ through the molecule is recorded as a function of bias voltage $(V)$ and gate voltage $\left(V_{g}\right)$. (b) (Top) $d I / d V$ maps at zero magnetic field (left) and at $8 \mathrm{~T}$ (right). At $0 \mathrm{~T}$, no SET lines corresponding to transitions between the ground states of $N$ and $N-1$ are present, as a result of ground-state spin blockade. At $8 \mathrm{~T}$, these lines do appear and the blockade is lifted. (Bottom) $d I / d V$ traces at zero bias, showing a charge degeneracy peak at $8 \mathrm{~T}$ and its complete suppression at $0 \mathrm{~T}$.

bias. This asymmetry suggests that the corresponding excitation energy is influenced by the bias voltage. The region $N-1$ also contains a zero-bias line, which resembles a Kondo resonance [28].

The striking difference between the maps at 0 and $8 \mathrm{~T}$ in Fig. 1(b) is the presence of the charge degeneracy point; the complete suppression of this point at $0 \mathrm{~T}$ is completely lifted at $8 \mathrm{~T}$. To study the blockade mechanism in more detail, we show two $d I / d V$ maps in a larger gate and 

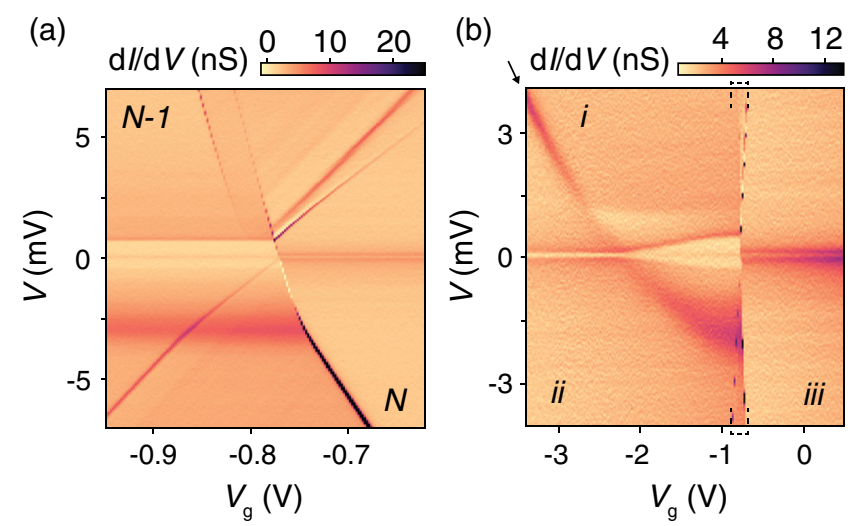

FIG. 2. $d I / d V$ maps at $0 \mathrm{~T}$ in a wide bias- and gate-voltage range. (a) $d I / d V$ map showing the same features as in Fig. 1(b), along with a ground-state transition line at $V=-3 \mathrm{mV}$ and additional SET excitation lines with different slopes. The SET lines at negative bias change slope at the ground-state transition. (b) $d I / d V$ map in a larger gate window, where the region marked by dashed brackets is the region shown in (a). The dark parabolic feature indicated by the arrow is the ground state transition line in (a). Three different regions with different ground states are separated by the discontinuity and the parabola, labeled (i), (ii), and (iii). The excitation energy of the COT excitations in (i) are tunable by the gate voltage.

bias-voltage range in Fig. 2, both recorded at $0 \mathrm{~T}$. In Fig. 2(a), the same features as in Fig. 1(b) can be observed, along with a broad horizontal line at $V=-3 \mathrm{mV}$ and additional SET excitation lines at higher bias. The Coulomb edges at negative bias change slope at the two coincidences with the broad horizontal line. This feature shows striking similarities with the simulated transport data presented in Ref. [29], where a singlet-triplet ground-state transition line in a double-quantum-dot model is studied. The energies of the ground states separated by this line have a different dependence on bias voltage, causing their corresponding Coulomb edges to have different slopes.

The gate- and bias-voltage dependence of the groundstate transition line is shown in Fig. 2(b) where the transport data are presented for an even wider gate-voltage range. The dashed brackets in this figure mark the gate-voltage range of Fig. 2(a). Figure 2(b) reveals that the ground-state transition line, indicated by the arrow, extends towards positive-bias voltages and has an anomalous parabolic shape (see Supplemental Material for a more detailed analysis [23]). Three regions with different ground states can be distinguished, separated by the parabola and the Coulomb edges. We label the regions (i)-(iii), where (i) and (ii) belong to the charge state $N-1$, and (iii) belongs to the charge state $N$. In both (ii) and (iii), a pair of COT lines appears at $V= \pm 0.1 \mathrm{mV}$, symmetrically positioned around zero bias. In (i), a pair of gate-voltage dependent COT lines is present, starting at $V= \pm 0.1 \mathrm{mV}$ around $V_{g}=-2 \mathrm{~V}$, moving away from zero bias as $V_{g}$ is increased. The excitation energy of this COT line thus depends on the gate voltage.
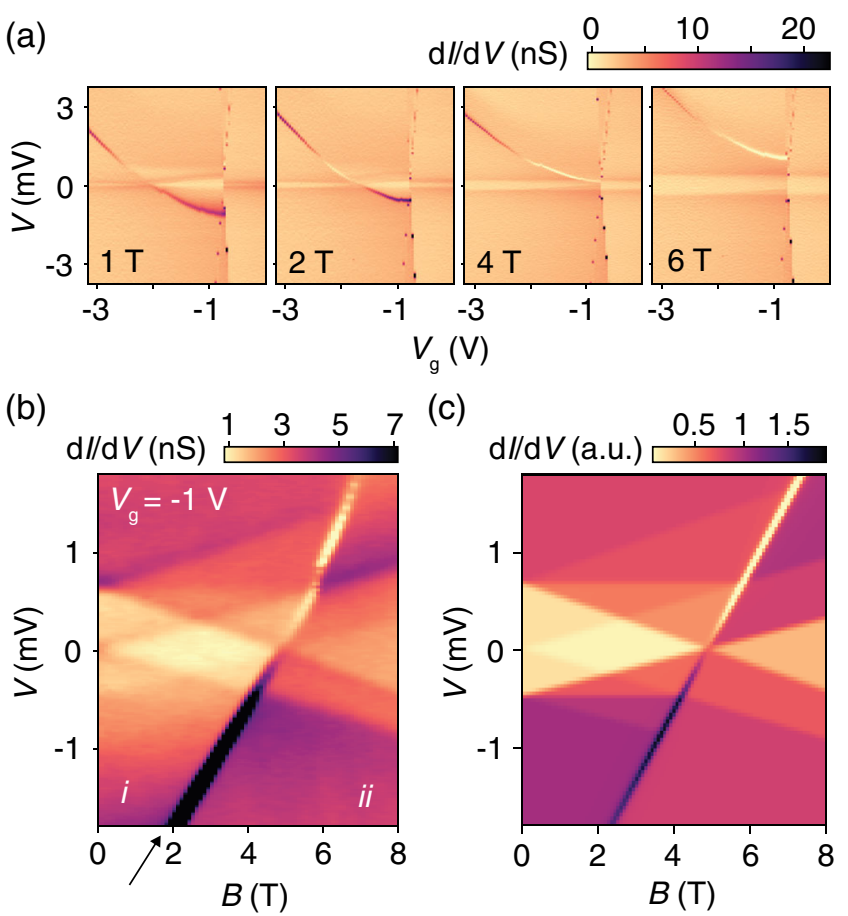

FIG. 3. (a) $d I / d V$ maps of the same region as Fig. 2(b), at different magnetic fields. The parabolic line separating the two spin ground states in $N-1$ moves upwards by increasing the magnetic field as the high-spin state becomes energetically more favorable. (b) Magnetic field dependence of the $d I / d V$ spectrum at $V_{g}=-1.0 \mathrm{~V}$. One line originating from the excited multiplet crosses zero bias at $B=5 \mathrm{~T}$ and becomes the new ground state above this field. The two ground states are separated by the slanted line indicated by the arrow. (c) Simulated $d I / d V$ spectra for a system of a spin- $1 / 2$, tunnel coupled to two reservoirs, and exchange coupled to a spin-3/2, with a $V$-dependent exchange coupling.

Next, we investigate the magnetic-field dependence of the parabolic ground-state transition line. Figure 3(a) shows four maps in the same bias- and gate-voltage range as in Fig. 2(b), at different magnetic fields. By increasing the magnetic field, the parabola moves towards higher bias voltages, and the $d I / d V$ along the line changes in magnitude and sign. For an increasing part of the line, the $d I / d V$ turns negative; i.e., the current goes down by increasing the bias voltage at the transition from (ii) to (i).

The fact that the ground-state transition line moves upwards with magnetic field implies that the total spin of the ground state in region (ii) is larger than in region (i); upon increasing the magnetic field, the ground state with higher spin becomes energetically more favorable and the transition occurs at higher bias voltages. Above $4 \mathrm{~T}$, region (ii) moves across zero bias at the charge degeneracy point, which lifts the GSSB at this point. The parabola thus marks the transition from a spin-blockaded region (i), to a region where the blockade is lifted (ii).

To identify the spin states in $N-1$, we focus on the magnetic-field dependence of the $d I / d V$ spectrum in $N-1$ 
(see Supplemental Material [23] for a discussion on the spectrum of charge state $N$, which includes Refs. [24-27]), recorded at a fixed gate voltage $V_{g}=-1 \mathrm{~V}$ [see Fig. 3(b)]. The Kondo-like peak at zero bias splits linearly in two in a magnetic field, which verifies the presence of a degenerate ground state at $0 \mathrm{~T}$. The COT excitations at $V=+0.7$ and $V=-0.4 \mathrm{mV}$ split in three and appear asymmetric in position and intensity. The fact that these excitations split in three implies that the corresponding excited state is a spin multiplet with a larger spin than the ground state; this can be deduced from the spin selection rules for COT processes [17]. Moreover, the spin selection rules impose additional constraints, which lead to the conclusion that the spin difference between the excited state and the ground state is one.

At about $5 \mathrm{~T}$, one excitation from the excited spin multiplet crosses zero bias, which at that point becomes the new spin ground state. The two regions with different ground states are separated by the slanted line indicated by the arrow. These regions correspond to (i) and (ii) in Fig. 2(b), labeled accordingly in Fig 3(b). The finite slope of the ground-state transition line results from the influence of the bias voltage on the associated excitation energy. This is also reflected by the asymmetry in bias voltage at which the multiplet excitations appear.

A model explaining the main features of the experimental data can now be constructed. The observed spin excitations occur at relatively low bias voltages $(\sim 1 \mathrm{mV})$, which suggests that they are not related to spin reconfigurations of the Mn center itself, as for spin-crossover molecules [30,31]; the energies of these transitions are typically orders of magnitude larger. Rather, we propose a model in which we invoke a weakly coupled spin, exchange coupled to the high-spin center. This spin is possibly residing on the ligands of the molecule, as in other molecular systems $[5,32]$ and we will refer to it as the ligand spin. We present a simulation of this system's spin-excitation spectra, based on Ref. [33] in Fig. 3(c) (see Supplemental Material for a description of the simulation [23]).

The ground state (GS) and excited state (ES) in the simulation of $N-1(N)$ are sketched on the left-hand (right-hand) side in Fig. 4, where red arrows represent the ligand spins, green arrows represent the spins of the $\mathrm{Mn}$ center, and yellow spheres represent the gold atoms of the electrodes. The observed SET and COT transitions between the states are indicated by the solid and dashed black arrows, respectively. In the transition from charge state $N-1$ to $N$, the added electron is likely to reside on the metal center, changing the oxidation state from $\mathrm{Mn}^{4+}$ to $\mathrm{Mn}^{3+}$, as illustrated in Fig. 4. This charge transition of the Mn center occurs in solutions of this compound, as shown by cyclic voltammetry measurements [22]. The spin of the Mn center in $N$ would then match the spin measured in crystals of this type of compound [34], namely $S=2$.

For a GSSB to occur in this system, the ground-state spin should change by more than $1 / 2$ upon charging. This can

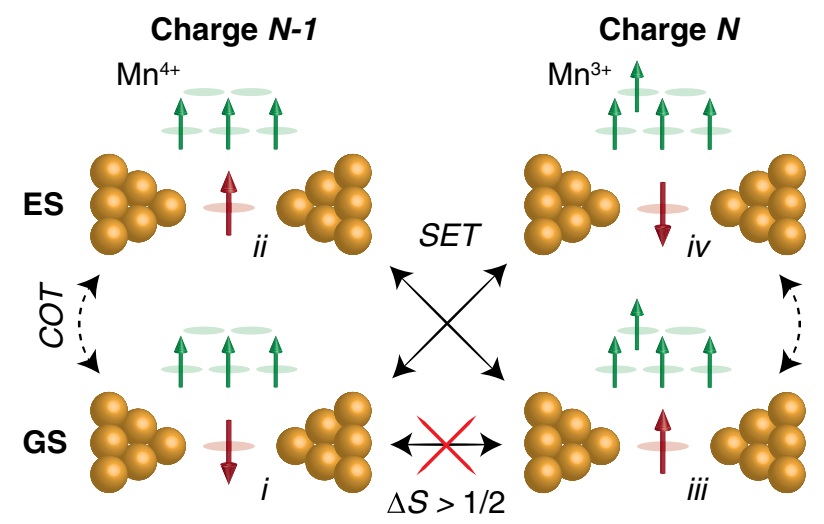

FIG. 4. Diagram showing the four states of different charge and spin, where the red and green arrows correspond to the ligand spin and the spins in the Mn center, respectively. The observed SET and COT transitions are represented by solid and dashed arrows, respectively. The red cross indicates the blocked SET transition. For charge state $N-1, S=1$ (i) and $S=2$ (ii), and for charge state $N, S=5 / 2$ (iii) and $S=3 / 2$ (iv).

happen if the added charge not only contributes with its intrinsic spin, but its presence also changes the sign of the exchange coupling from antiferromagnetic to ferromagnetic, further increasing the total spin by one. Such a transition of the exchange coupling is observed, and it is marked by the parabolic ground-state transition line in $N-1$. We performed a model calculation to show that for this molecular system there exists a realistic scenario under which a sign change of the exchange coupling occurs (see Supplemental Material [23]).

The total spin of states (i) and (iii), as shown in Fig. 4, are 1 and $5 / 2$, respectively, which amounts to a spin difference of $3 / 2$. SET transitions between these ground states are thus rendered forbidden, as indicated by the red cross in Fig. 4. At high magnetic fields, the high-spin state (ii) $(S=2)$ becomes the ground state for $N-1$, lowering the ground-state spin difference to $1 / 2$, whereby the GSSB is lifted.

This work shows that the act of charging a nanoscale object is not merely a consecutive filling of the lowest unoccupied orbitals, whereby the spin changes by $\pm 1 / 2$. Rather, the act of charging can have a strong influence on the internal exchange couplings of the object, to a degree that ground-state transitions cannot be realized by single charge carriers. The presence of a GSSB is not restricted to single-molecule systems: every nano-object with a spin $S>1$ can potentially host this effect under the right conditions. A thorough understanding of the GSSB is therefore of essence in research directions where high-spin devices are increasingly gaining interest, such as spintronics [1-3] and quantum computing [4-6].

In summary, we have demonstrated a GSSB in a singlemolecule junction, which can be reversibly lifted by driving the system through a magnetic ground-state transition with an external magnetic field. The blockade results from a sign 
change of the exchange coupling upon charging, which causes the ground-state spin of subsequent charge states to differ by more than $1 / 2$. This work demonstrates how the act of charging can induce a magnetic phase transition in a high-spin device by which resonant transport is completely suppressed.

This work was supported by the Netherlands Organisation for Scientific Research (NWO/OCW), as part of the Frontiers of Nanoscience program, and the ERC Advanced Grants No. 240299 (Mols@Mols) and No. 788822 (Mol-2D). P. G. acknowledges a Marie Skłodowska-Curie Individual Fellowship under Grant TherSpinMol (ID: 748642) from the European Union's Horizon 2020 research and innovation programme. The work in Spain is supported by the Spanish MINECO (Unit of Excellence Maria de Maeztu MDM2015-0538 and Project MAT2017-89993-R cofinanced by the Fondo Europeo de Desarrollo Regional (FEDER)) and the Generalitat Valenciana (PROMETEO Programme). The Center for Quantum Devices is funded by the Danish National Research Foundation.

*H.S.J.vanderZant@tudelft.nl

[1] L. Bogani and W. Wernsdorfer, in Nanoscience and Technology: A Collection of Reviews from Nature Journals (World Scientific, Singapore, 2010), pp. 194-201.

[2] S. Sanvito, Chem. Soc. Rev. 40, 3336 (2011).

[3] J. M. Clemente-Juan, E. Coronado, and A. Gaita-Ariño, Chem. Soc. Rev. 41, 7464 (2012).

[4] M. N. Leuenberger and D. Loss, Nature (London) 410, 789 (2001).

[5] S. Thiele, F. Balestro, R. Ballou, S. Klyatskaya, M. Ruben, and W. Wernsdorfer, Science 344, 1135 (2014).

[6] M. Shiddiq, D. Komijani, Y. Duan, A. Gaita-Ariño, E. Coronado, and S. Hill, Nature (London) 531, 348 (2016).

[7] F. H. L. Koppens, J. A. Folk, J. M. Elzerman, R. Hanson, L. H. W. Van Beveren, I. T. Vink, H.-P. Tranitz, W. Wegscheider, L.P. Kouwenhoven, and L. M. K. Vandersypen, Science 309, 1346 (2005).

[8] A. C. Johnson, J. R. Petta, C. M. Marcus, M. P. Hanson, and A. C. Gossard, Phys. Rev. B 72, 165308 (2005).

[9] H. W. Liu, T. Fujisawa, Y. Ono, H. Inokawa, A. Fujiwara, K. Takashina, and Y. Hirayama, Phys. Rev. B 77, 073310 (2008).

[10] J. Koch and F. von Oppen, Phys. Rev. Lett. 94, 206804 (2005).

[11] R. Leturcq, C. Stampfer, K. Inderbitzin, L. Durrer, C. Hierold, E. Mariani, M. G. Schultz, F. Von Oppen, and K. Ensslin, Nat. Phys. 5, 327 (2009).

[12] E. Burzurí, Y. Yamamoto, M. Warnock, X. Zhong, K. Park, A. Cornia, and H. S. J. van der Zant, Nano Lett. 14, 3191 (2014).
[13] C. B. Winkelmann, N. Roch, W. Wernsdorfer, V. Bouchiat, and F. Balestro, Nat. Phys. 5, 876 (2009).

[14] M. T. Deng, S. Vaitiekènas, E. B. Hansen, J. Danon, M. Leijnse, K. Flensberg, J. Nygård, P. Krogstrup, and C. M. Marcus, Science 354, 1557 (2016).

[15] D. Weinmann, W. Häusler, and B. Kramer, Phys. Rev. Lett. 74, 984 (1995).

[16] H. B. Heersche, Z. de Groot, J. A. Folk, H. S. J. van der Zant, C. Romeike, M. R. Wegewijs, L. Zobbi, D. Barreca, E. Tondello, and A. Cornia, Phys. Rev. Lett. 96, 206801 (2006).

[17] R. Gaudenzi, M. Misiorny, E. Burzurí, M. R. Wegewijs, and H. S. J. van der Zant, J. Chem. Phys. 146, 092330 (2017).

[18] L. P. Rokhinson, L. J. Guo, S. Y. Chou, and D. C. Tsui, Phys. Rev. B 63, 035321 (2001).

[19] A. K. Hüttel, H. Qin, A. W. Holleitner, R. H. Blick, K. Neumaier, D. Weinmann, K. Eberl, and J. P. Kotthaus, Europhys. Lett. 62, 712 (2003).

[20] H. Park, A. K. L. Lim, A. P. Alivisatos, J. Park, and P. L. McEuen, Appl. Phys. Lett. 75, 301 (1999).

[21] E. A. O’Neill, K Osorio, and H. S. J. Van der Zant, Appl. Phys. Lett. 90, 133109 (2007).

[22] C. Allain, S. Favette, L.-M. Chamoreau, J. Vaissermann, L. Ruhlmann, and B. Hasenknopf, Eur. J. Inorg. Chem. 2008, 3433 (2008).

[23] See Supplemental Material at http://link.aps.org/ supplemental/10.1103/PhysRevLett.122.197701 for a description of the synthesis and the properties of the molecule, which includes Refs. [24-27].

[24] E. Burzurí, R. Gaudenzi, and H. S. J. van der Zant, J. Phys. Condens. Matter 27, 113202 (2015).

[25] S. Herzog and M. R. Wegewijs, Nanotechnology 21, 274010 (2010).

[26] W. G. van der Wiel, S. De Franceschi, J. M. Elzerman, S. Tarucha, L. P. Kouwenhoven, J. Motohisa, F. Nakajima, and T. Fukui, Phys. Rev. Lett. 88, 126803 (2002).

[27] M. Pustilnik and L. I. Glazman, Phys. Rev. Lett. 87, 216601 (2001).

[28] W. Liang, M. P. Shores, M. Bockrath, J. R. Long, and H. Park, Nature (London) 417, 725 (2002).

[29] C. Stevanato, M. Leijnse, K. Flensberg, and J. Paaske, Phys. Rev. B 86, 165427 (2012).

[30] T. Miyamachi, M. Gruber, V. Davesne, M. Bowen, S. Boukari, L. Joly, F. Scheurer, G. Rogez, T. K. Yamada, P. Ohresser et al., Nat. Commun. 3, 938 (2012).

[31] V. Meded, A. Bagrets, K. Fink, R. Chandrasekar, M. Ruben, F. Evers, A. Bernand-Mantel, J. S. Seldenthuis, A. Beukman, and H. S. J. van der Zant, Phys. Rev. B 83, 245415 (2011).

[32] E. A. Osorio, K. Moth-Poulsen, H. S. J. van der Zant, J. Paaske, P. Hedegård, K. Flensberg, J. Bendix, and T. Bjørnholm, Nano Lett. 10, 105 (2010).

[33] M. Ternes, New J. Phys. 17, 063016 (2015).

[34] A. Abhervé, M. Palacios-Corella, J. M. Clemente-Juan, R. Marx, P. Neugebauer, J. van Slageren, M. Clemente-León, and E. Coronado, J. Mater. Chem. C 3, 7936 (2015). 